\title{
Causes \& Management of Testicular Abscess: Findings of A Study on Eleven Patients
}

\author{
Subikas Biswas ${ }^{1}$, Gandhari Basu ${ }^{2}$ \\ 1(Surgery, College of Medicine \& JNM Hospital, WBUHS, India) \\ 2(Community medicine, College of Medicine \& JNM Hospital, WBUHS, India)
}

\begin{abstract}
Testicular abscess is a rare surgical entity of Zone III of inguinoscrotal region. C Trachomatis and E Coli are the two commonest causative organism worldwide. A total number of eleven cases of testicular abscesses had been reviewed for a period of one and half years. It was seen that, the cases mainly present with complaint of acute scrotum with discharging pus and acute abdomen. Mean age of the patients under study is around 53 yrs. Ultrasonography used for diagnosis. In two third cases epididymoorchitis history was obtained. Regarding growth pattern of pus, it was found that identifying bacterial pathogens are found in seven case where as no growth was found in rest. E coli was found to be the commonest pathogen in culture isolate. One patient of chronic testicular abscess, who was on treatment for pulmonary tuberculosis; pus culture revealed AFB to be causative bacteria. Only a single case of acute testicular abscess was found to have seropositivity for $H I V$ virus, his pus culture was notably negative for any other bacterial pathogen. One out of the 7 cases of acute testicular abscess and 2 of the 4 cases of chronic testicular abscess were found to have type II DM. Orchiectomy and broad spectrum antibiotics were used as mainstay of treatment.
\end{abstract}

Keywords: AFB, acute abdomen, epididymoorchitis, inguinoscrotal region, orchiectomy, testicular abscess, type II DM, ultrasonography.

\section{Introduction}

Testicular abscess is a rare relatively unenchanted surgical entity of Zone III of inguinoscrotal region ${ }^{1}$. It is generally preceded by epididymoorchitis. Epididymoorchitis is most often due to the retrograde extension of organisms from the vas deferens and is rarely the result of haematogenous spread.The causative organism is identified in $80 \%$ of patients and varies according to the age of the patient. In prepubertal and old age groups coliforms (E.Coli) are considered to be commonest pathogen while in sexually active age group, C.Trachomatis is the commonest bacteria identified. A multitude of other factors causing testicular abscess are also identified namelymumps and some other viral infections, testicular trauma, testicular infarction, chemicals,secondary to brachytherapy to prostate and rarely following ruptured appendicitis. ${ }^{2,3} \mathrm{In}$ immune compromised patients, testicular abscess may result from tuberculosis or fungal infection. ${ }^{4}$ Signs and symptoms of testicular abscess usually include unilateral testicular pain, swelling and scrotal erythema with a palpable tender mass on examination. Ultrasound is particularly helpful in making the diagnosis showing a heterogeneous hypoechoic focus with a hyperemic rim. ${ }^{2,5}$ Treatment of testicular abscess consists of appropriate antibiotics alone or with surgical drainage if necessary. ${ }^{6}$ Uncommonly, severe cases may necessitate orchiectomy. ${ }^{7}$

\section{Case Particulars And Proceedings}

Between 2009 and 2010, four cases of acute scrotum were undergone ultrasonography. Three of them were diagnosed to have testicular abscess (Fig.1a \&Fig.1b). The remaining one had heterogenous Hypoechoic lesion in testis (Fig.2). That patient had a previous history of trauma to scrotum two days back and was provisionally diagnosed to have haematocele of testis. Those whowere diagnosed to have testicular abscess were admitted, treated with antibiotics as appropriate and drainage of pus. Postoperatively they failed to respond to conservative treatment as projected from their clinical status and laboratory results even after 5 days and decision of orchidectomy was taken. Following orchidectomy all of them responded positively and wasdischarged at median period of 6.6days (range $4^{\text {th }} \cdot 10^{\text {th }}$ day)postoperatively. Patient who had haematocele of testis was treated with antibiotic, other accessory medications and measures as appropriate but admitted on second day with high fever and raised ESR. He failed to respond to intravenous broad spectrum antibiotics on admission even after 48 hours and was undergone orchidectomy. During orchidectomy his testis was found to have filled with thick pus and altered blood rupturing into the scrotum. He responded quickly postoperatively and was discharged on $8^{\text {th }}$ postoperative day.

One case was presented with acute abdomen having obstructed right inguinal hernia. He had a history suggestive of reducible right inguinal hernia that had got obstructed. He was undergone ultrasonography of whole abdomen and of inguinoscrotal region by his family physician. Ultrasonography of his inguinoscrotal region revealed obstructed intestine along with a hypo echoic lesion in ipsilateral testis. Inguinoscrotal 
exploration was undertaken. On exploration a loop of ileum was found; it was returned to abdomen after checking its viability. During operation the testis was found to be filled with pus with no viable testicular tissue. Orchidectomy was undertaken. Internal inguinal ring was closed followed by herniorrhaphy.

Two cases presented with acute scrotum with sonologically diagnosed Hypoechoic lesion in testis and reduced blood flow in Doppler.(Fig.3 \& Fig.4) All of them were treated by local doctors for epididymoorchitis and urinary tract infection with antibiotics for four weeks. Among these two patients one was previously diagnosed to be seropositive for human immunodeficiency virus; another had a history of undergoing catheterization by local doctor for treating acute retention of urine. Exploration of scrotum was undertaken in both cases and orchidectomy was done as there was no viable testicular tissue (Fig.5a \& Fig.5b).

Between 2009 and 2010 four other patients were presented with a discharging sinus of scrotum draining plenty of pus with protruding testicular soft tissue through the opening of the sinus (Fg.6) and were treated with orchidectomy with excision of diseased scrotal skin. Among them two patients were on oral antidiabetic medications for about last 8 years for type II DM. They were on treatment with various antibiotics for a mean period of 21.6 weeks (range - 19wks to 23 wks.). One patient was in treatment with four drugs regimen for pulmonary tuberculosis for 2 months.

\section{Results}

In our study period thus total 11 patients of testicular abscess were treated with orchidectomy were studied. Among these, 7 patients $(63.63 \%)$ were suffering from acute testicular abscess and $4(36.36 \%)$ from chronic testicular abscess. Clinical records, demographical factors of all the patients were reviewed meticulously and also the results of microbiological examination and culture sensitivity reports of the pus. Routinely all orchidectomy specimen were sent for histopathological examination.

Mean age of the patients under study is 53.18 yrs (range $-28-72$ yrs) while the mean age of patients presented with acute testicular abscess and chronic testicular abscess are 48.85yrs (range28 - 62)and $60.5 \mathrm{yrs}$ (range $-40-72 \mathrm{yrs}$ ) respectively. No specific age group was found to have statistical predilection for development of any of the two forms of testicular abscess. About $63.63 \%$ of the patients gave history of epididymoorchitis recently or in past before development of testicular abscess.(table 1)

Regarding growth pattern of pus (table 2) it is found that identifying bacterial pathogens are found in $63.63 \%$ of cases where as no growth is observed in $36.36 \%$ of cases. In the former E coli is found to be the commonest bacteria in culture isolate. $50 \%$ of the patients of chronic testicular abscess and $28.56 \%$ of acute testicular abscess showed no growth in their culture isolate. One patient of chronic testicular abscess, who was on treatment for pulmonary tuberculosis; pus culture revealed AFB to be causative bacteria. Only a single case of acute testicular abscess was found to have seropositivity for HIV virus, his pus culture was notably negative for any bacterial pathogen. One (14.28\%) of the 7 cases of acute testicular abscess and $2(50 \%)$ of the 4 cases of chronic testicular abscess are found to have type II DM.

\section{Discussion}

Very few literatures are there to deal with etiopathology, clinical features and treatment standards of testicular abscess masking it really an enchanted surgical entity. Incidence rate of testicular abscess is less than 1 per 1000 male per year. Inguinoscrotal composite unit has been divided into four surgical zones by S Khan et al ${ }^{1}$ for better description of disease processes of the inguinoscrotal region. Testicular abscess is a rare surgical disorder of zone III that may present with acute scrotum.

Testicular abscess is usually occurred as a consequence of unresolved acute epididymoorchitis in which the causative pathogen infects the testis and it's covering by retrograde path via vas deferens. Rarely it may reach the testis via haematogenous route. A number of other causes of testicular abscess are also identified; namely mumps and other viral infections, testicular trauma, testicular infarction, chemicals, secondary to brachytherapy to prostate and rarely following ruptured appendicitis. ${ }^{2,3}$ In Immune compromised patients, testicular abscess may result from tuberculosis or fungal infection. ${ }^{4}$

Classification of acute and chronic testicular abscess in our study population is done depending on clinical presentation of the disease process. Most of the patients with acute testicular abscess presented with acute scrotum and required prompt surgical intervention. On the contrary chronic testicular abscess lacks symptoms or signs of acuteness. They fail to resolve completely on initial incision and drainage of acute testicular abscess. Initially their signs and symptoms resolved but eventually they pass to the phase of chronicity. Even after six weeks of time period their wound fail to heal and they presented with discharging scrotum with a sinus draining pus and soft tissue debris.

Classically testicular abscess is treated by appropriate antibiotics alone or with surgical drainage if necessary ${ }^{6}$. If a conservative approach is attempted (i.e.medical therapy) then serial ultrasound examinations should be performed to ensure that the abscess does not progress. Severe cases may necessitate orchiectomy ${ }^{7}$. Chronic testicular abscess usually require orchidectomy as in most cases no viable testicular tissue could be 
obtained $^{7}$.In our series we have found that commonest cause of testicular abscess is epididymoorchitis but the commonest isolate from the pus is EColi contrary to the $\mathrm{C}$ Trachomatis as found in various larger trials. ${ }^{8}$ However this result corroborates with the experience of Granados Loarca et al. ${ }^{6}$

Interestingly in our series mean age of the patients are $48.85 \mathrm{yrs}, 60.5 \mathrm{yrs}$ for acute and chronic testicular abscess respectively with a mean age of overall study population being 53.18 yrs and all are contrary to lower mean age of presentation with testicular abscess ${ }^{9}$. Difference of age distribution of our series with those of the different trials could be due to use of effective treatment at diagnosis, use of effective new generation broad spectrum antibiotics and may be as a result of sample selection from different sociocultural population and geographical location.

Chronic testicular abscess has more chance to be negative in culture result and patients of chronic testicular abscess has more association with type II DM than acute testicular abscess although in this small series of cases no statistical association could be proved. Association of type II DM more with chronic testicular abscess could be accredited due to higher mean age of presentation of the disease in patients with chronic testicular abscess.

\section{Figures:}

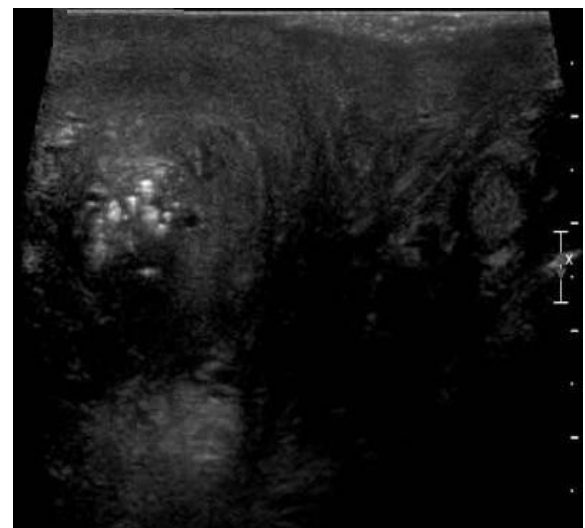

Fig.1a

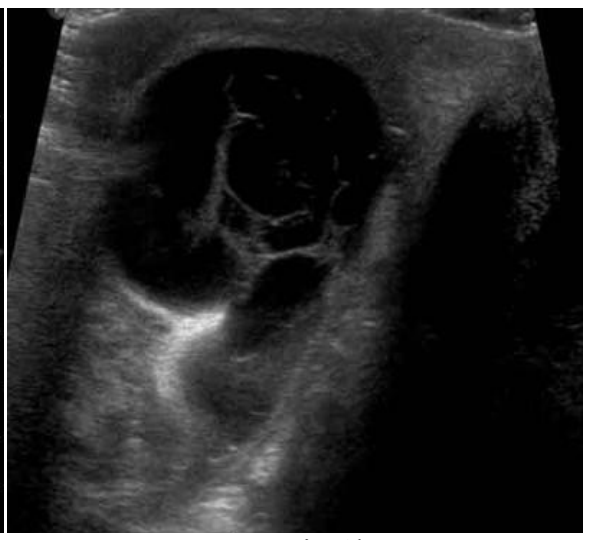

Fig.1b

Fig.1a\& Fig.1b: Ultrasonography of testicular abscess in two different patients presenting with acute scrotum

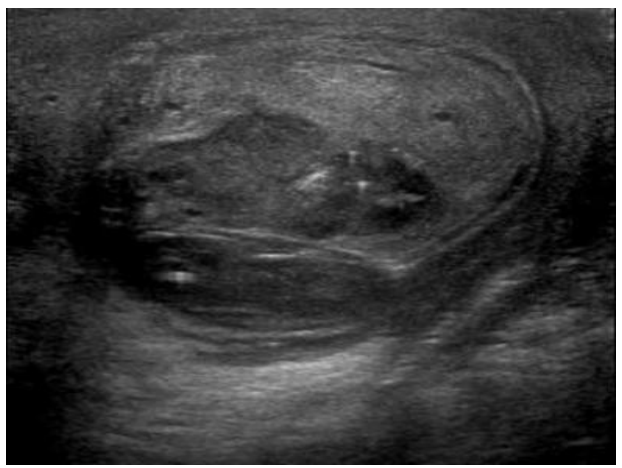

Fig.2: Ultrasonography of rt. testis showing haematocele

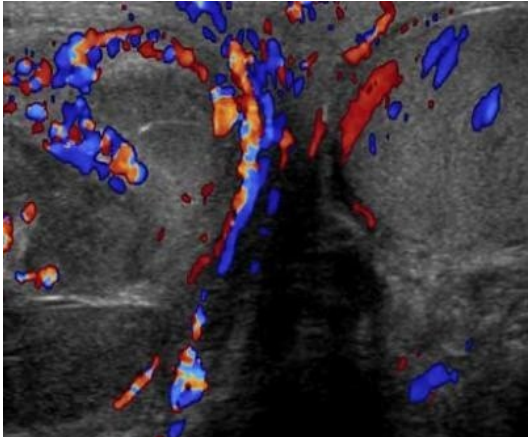

Fig.3

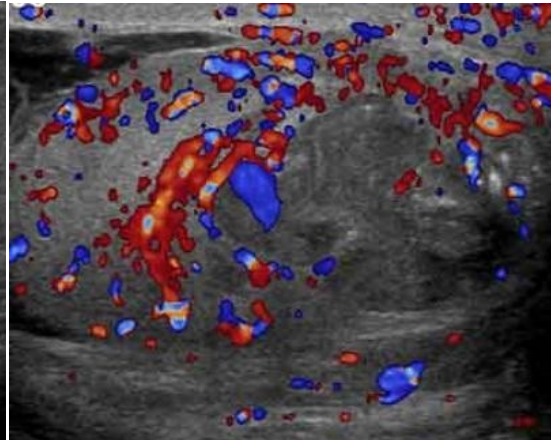

Fig.4

Fig.3 \& Fig.4: Color Doppler sonography of testis and scrotum showing hypoechoic lesion in testis and reduced blood flow in testis suggestive of testicular abscess. 


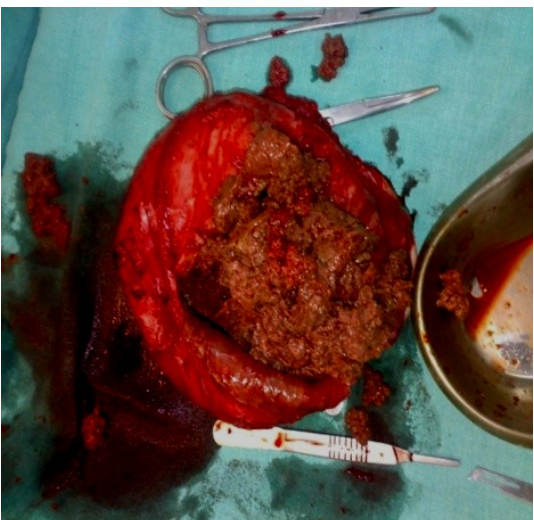

Fig.5a

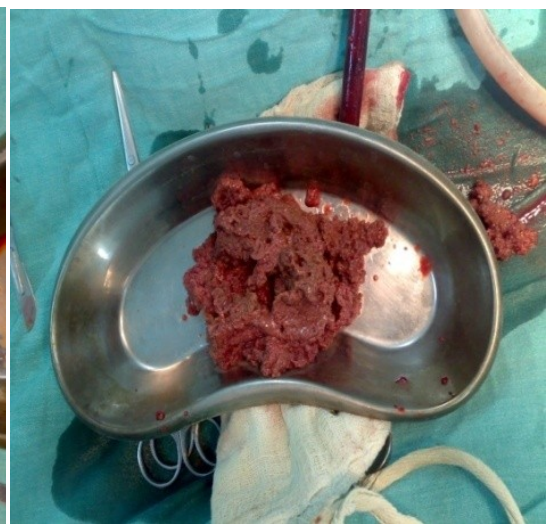

Fig.5b

Fig.5a: Showing no viable testicular tissue on exploration

Fig.5b: Dead testicular tissue converted into a brown spongy leathery mass

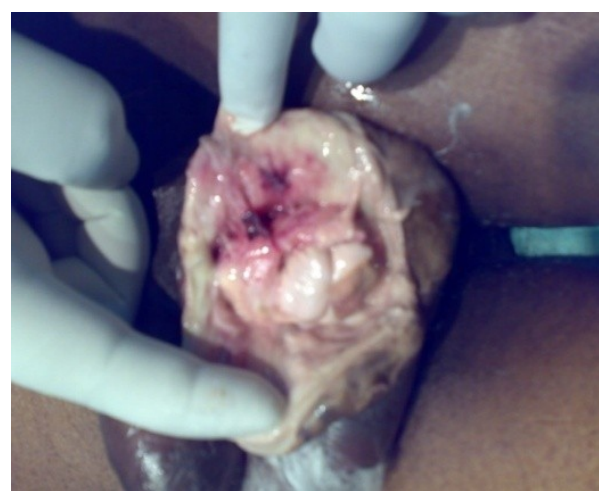

Fig.6: Patient presenting with chronic discharging sinus of scrotum with protruding testicular soft tissue and pus coming out through the sinus tract

\section{TABLES:}

Table1: Causes of testicular abscess $(\mathbf{n}=11)$

\begin{tabular}{|lll|}
\hline Cause & Number & Percentage (\%) \\
Epididymoorchitis & 7 & 63.63 \\
Traumatic (following infected haematocele) & 1 & 9.09 \\
Postinstrumentation \& catheterization & 1 & 9.09 \\
Systemic tuberculosis & 1 & 9.09 \\
Obstructed hernia & 1 & 9.09 \\
\hline
\end{tabular}

Table 2: Growth pattern in pus from patients of testicular abscess $(n=11)$

\begin{tabular}{|lll|}
\hline Growth characteristics & Number & Percentage (\%) \\
E coli & 4 & 36.36 \\
Noncoliforms & 3 & 27.27 \\
Streptococcus & 1 & \\
Pseudomonas & 1 & 9.09 \\
Acid fast bacillus & 1 & 9.09 \\
No growth & 4 & 9.09 \\
\hline
\end{tabular}

\section{Conclusion}

Testicular abscess is most commonly resulted following a sequel of unresolved epididymoorchitis where the infection spread to involve the testis. Classification of acute and chronic testicular abscess that we have used in our study is an arbitrary one. However from treatment purpose its significance could be portrayed as acute testicular abscess requires orchidectomy when it is severe enough. On the contrary chronic testicular abscess more often demands orchidectomy as in most of the cases no viable testicular tissue remained left. We have studied the demography of the disease process and found that the mean age of presentation of testicular abscess and both of its subdivisions are more than what is said in classical teaching. However we still recommend a more detailed study of the disease process as no statistical remarks could be brought out with this small study population. 


\section{Acknowledgements}

We sincerely express our gratitude to members of microbiological laboratory and all those patients and their family members besides whom this study could never be completed.

\section{References}

[1]. S. Khan, J. Rehman, B. Chughtai, D. Sciullo, E. Mohan \& H. Rehman : Anatomical Approach to Scrotal Emergencies: A New Paradigm for the Diagnosis and Treatment of the Acute Scrotum. The Internet Journal of Urology. 2010 Volume 6 Number 2

[2]. Dogra, V.S., et al., Sonography of the scrotum. Radiology, 2003. 227(1): p. 18.36.

[3]. Thakur, A., et al., Scrotal abscess following appendectomy. PediatrSurgInt, 2001. 17(7): p. 569.71

[4]. Granados Loarca, E.A., [Testicular abscess: a manifestation of tuberculosis]. ActasUrolEsp, 1998. 22(4): p. 381.3.

[5]. Farriol, V.G., et al., Gray.scale and power dopplersonographic appearances of acute inflammatory diseases of the scrotum. J Clin Ultrasound, 2000. 28(2): p. 67.72

[6]. Granados Loarca, E.A. et al. Epididymo.testicular abscess. Arch EspUrol, 1994. 47(6): p. 553.6.

[7]. Slavis, S.A., J. Kollin, and J.B. Miller, Pyocele of scrotum: consequence of spontaneous rupture of testicular abscess. Urology, 1989. 33(4): p. 313.6.

[8]. Trojian TH, Lishnak TS, Heiman D (April 2009). "Epididymitis and orchitis: an overview". Am Fam Physician79 (7): $583-7$.

[9]. Schlossberg D. Clinical Infectious Disease. Cambridge Univ Pr. (2008) 